\title{
Adverse Event Severity Code
}

National Cancer Institute

\section{Source}

National Cancer Institute. Adverse Event Severity Code. NCI Thesaurus. Code $C 93721$.

A coded value specifying the intensity of the event. 